\title{
BMJ Open Strategies to reduce antibiotic use in women with uncomplicated urinary tract infection in primary care: protocol of a systematic review and meta- analysis including individual patient data
}

\author{
Judith Heinz (1) , ${ }^{1}$ Christian Röver, ${ }^{1}$ Ghefar Furaijat, ${ }^{2,3}$ Yvonne Kaußner, ${ }^{4}$ \\ Eva Hummers, ${ }^{2}$ Thomas Debray, ${ }^{5}$ Alastair D Hay, ${ }^{6}$ Stefan Heytens, ${ }^{7}$ \\ Ingvild Vik (D) , 8,9 Paul Little (D) , ${ }^{10}$ Michael Moore (D) , ${ }^{10}$ Beth Stuart, ${ }^{10}$ \\ Florian Wagenlehner, ${ }^{11}$ Andreas Kronenberg, ${ }^{12}$ Sven Ferry, ${ }^{13}$ Tor Monsen, ${ }^{13}$ \\ Morten Lindbaek, ${ }^{8}$ Tim Friede (D) , ${ }^{1}$ Ildiko Gagyor (D) ${ }^{4}$
}

To cite: Heinz J, Röver C, Furaijat G, et al. Strategies to reduce antibiotic use in women with uncomplicated urinary tract infection in primary care: protocol of a systematic review and metaanalysis including individual patient data. BMJ Open 2020;10:e035883. doi:10.1136/ bmjopen-2019-035883

- Prepublication history and supplemental material for this paper are available online. To view these files, please visit the journal online (http://dx.doi. org/10.1136/bmjopen-2019035883).

$\mathrm{JH}$ and CR contributed equally.

Received 20 November 2019 Revised 29 May 2020

Accepted 16 July 2020

\section{Check for updates}

(C) Author(s) (or their employer(s)) 2020. Re-use permitted under CC BY-NC. No commercial re-use. See rights and permissions. Published by BMJ.

For numbered affiliations see end of article.

Correspondence to Dr Judith Heinz; judith.heinz@med.unigoettingen.de

\section{ABSTRACT}

Introduction Uncomplicated urinary tract infection (UTI) in women is a common reason to present in general practice and is usually treated with antibiotics to reduce symptom severity and duration. Results of recent clinical trials indicate that non-antibiotic treatment approaches can also be effective. However, it remains unclear which patients would benefit from antibiotic treatment and which can effectively and safely be treated without antibiotics. This systematic review and meta-analysis aims to estimate the effect of treatment strategies to reduce antibiotic use in comparison with immediate antibiotic treatment and to identify prognostic factors and moderators of treatment effects. A further aim is to identify subgroups of patients benefiting from a specific therapy.

Methods and analysis A systematic literature search will be performed to identify randomised controlled trials which investigated the effect of treatment strategies to reduce antibiotic use in female adults with uncomplicated UTI compared with immediate antibiotic treatment. Therefore, the primary outcome of the meta-analysis is incomplete recovery. Anonymised individual patient data (IPD) will be collected. Aggregate data will be used for pairwise comparisons of treatment strategies using meta-analysis models with random effects accounting for potential between-study heterogeneity. Potential effect moderators will be explored in meta-regressions. For IPD, generalised linear mixed models will be used, which may be adjusted for baseline characteristics. Interactions of baseline variables with treatment effects will be explored. These models will be used to assess direct comparisons of treatment, but might be extended to networks.

Ethics and dissemination The local institutional review and ethics board judged the project a secondary analysis of existing anonymous data which meet the criteria for waiver of ethics review. Dissemination of the results will be via published scientific papers and presentations. Key

\section{Strengths and limitations of this study}

This is the first individual patient data (IPD) metaanalysis to evaluate the effectiveness of experimental strategies to reduce antibiotic use in women with uncomplicated urinary tract infection in comparison with immediate antibiotic treatment.

- A clinical prognostic model will be developed in order to identify women who can be treated without (immediate) antibiotics effectively and safely and to facilitate an individualised treatment approach.

- The use of IPD will allow for detailed modelling aimed towards personalised treatment recommendations.

- The joint analysis of data from several studies will enhance the external validity of findings and the assessment thereof.

- The set of included studies may be subject to selection biases (eg, reporting bias, data availability bias) and may be heterogeneous in design, study population and intervention used.

messages will be promoted for example, via social media or press releases.

PROSPERO registration number CRD42019125804.

\section{INTRODUCTION}

Uncomplicated urinary tract infection (UTI) is a common condition affecting mostly women with a lifetime prevalence of $50 \%$ and is a common reason for women to present to general practice. ${ }^{12}$ Generally, primary care guidelines recommend immediate antibiotics as a first-line treatment. ${ }^{3}$ Consequently, UTI is among the most common causes for antibiotic prescription in primary care. ${ }^{4-8}$ 
Therefore, efforts to reduce their prescription are highly appreciated to reduce levels of antibiotic resistance. Physicians tend to overestimate the need and demand for antibiotics, and to prescribe them rather liberally although many women prefer non-antibiotic strategies to treat UTI if this is reliable and safe. ${ }^{259}$ Furthermore, the selective effect of antibiotics on antimicrobial resistance increasingly forces physicians to prescribe second-line antibiotics. ${ }^{10}$ Non-antibiotic treatment approaches as well as delayed prescription of antibiotics for UTI were tested in several randomised controlled trials (RCTs) showing reduced antibiotic use, but also delayed resolution of symptoms. ${ }^{11-16}$ In most of these trials, the chosen strategies could substantially reduce the number of antibiotic courses in women with uncomplicated UTI, but this was traded off by a somewhat higher symptom severity or longer symptom duration and a few more cases of febrile UTI, worsening symptoms or pyelonephritis. Overall, the results of the trials suggest that symptomatic treatment is effective, but it may not be the method of choice for every woman with uncomplicated UTI. Thus, reducing antibiotic prescribing can be impeded by uncertainty regarding which patients with UTI symptoms would benefit from antibiotic treatment and which ones can effectively and safely be treated without (immediate) antibiotics.

There is a lack of guidance as to when immediate antibiotic treatment may be appropriate, and when alternative measures may be adequate. Physicians usually use symptoms, signs and a point-of-care test (mostly dipsticks) to diagnose UTI, but predicting the outcome of uncomplicated UTIs will enable individualised treatment recommendations.

Therefore, the meta-analysis aims to (1) estimate the effect of experimental strategies to reduce antibiotic use in women with uncomplicated UTI compared with immediate antibiotic treatment as standard care; (2) identify moderators that modify treatment effects comparing experimental strategies with immediate antibiotic treatment and (3) identify prognostic factors at baseline associated with disease course of UTI in women allocated to experimental strategies and to develop a clinical prediction model to support treatment decisions in women with UTI.

\section{METHODS AND ANALYSIS}

In this systematic review and meta-analysis, RCTs comparing different strategies to reduce antibiotic use in women with uncomplicated UTI presenting to primary care will be identified and investigated. Individual patient data (IPD) will be collected to compare strategies aimed at reducing antibiotic use in women with uncomplicated UTI and immediate antibiotic treatment as a standard of care. If IPD are not available, aggregated results of the studies will at least be used for the estimation of treatment effects. The feasibility of a network meta-analysis will be investigated in order to allow for direct and indirect treatment comparisons. The present meta-analysis will be conducted in line with the Preferred Reporting Items for Systematic Reviews and Meta-Analyses (PRISMA) statement. ${ }^{17}$ The PRISMA checklist can be found in the online supplemental file 1 .

\section{Population}

The primary target population are female adults (aged 18 or older) with symptoms of an acute UTI presenting in general practice. In case results are stated only for mixed adult/adolescent populations, these will be considered as well, but paediatric populations (all younger than 18 years) will be excluded.

\section{Interventions}

The intervention is any experimental strategy to reduce antibiotic use (eg, symptomatic treatment with nonsteroidal anti-inflammatory drugs, herbal treatments, placebo or delayed antibiotic prescription).

\section{Controls}

The control is immediate antibiotic treatment as standard care.

\section{Outcomes}

Related to objectives (1) and (2) the primary outcome is incomplete clinical recovery; defined as more than slight symptoms (this applies to at least one of the scores for dysuria, frequency, urgency that were assessed last between days 3 and 7) at days 3-7 or occurrence of pyelonephritis, febrile UTI and sepsis or subsequent antibiotic treatment during a follow-up of at least 14 days. To assess the primary outcome with respect to UTI symptoms, we chose the time period of 3-7 days since most RCTs demonstrated the duration of moderately bad or worse symptoms within 3-4 days and a symptom resolution within 7 days in most of included patients. ${ }^{12151618}$ With respect to the criteria pyelonephritis and subsequent antibiotic treatment, we chose a follow-up period of at least 14 days. Uncomplicated UTI is a short condition and most RCTs followed up the patients for about 4 weeks. A longer follow-up period did not reveal changes regarding the complications and occurrence of recurrent UTI. ${ }^{19}$ For this reason and in order to include all available evidence on this topic, we chose a follow-up period of at least 14 days.

Secondary outcomes are antibiotic use (number of antibiotic courses) during a follow-up of at least 14 days, symptom burden at day 2, symptom burden at days 3-7, clinical recovery (defined as a symptom score of 0 for dysuria, frequency and urgency) at days 3-7 and recurrent UTI. Safety outcomes are complications (pyelonephritis, febrile UTI, sepsis) and (serious) adverse events within at least 14 days, related to the treatment or due to other reasons. Worsening of UTI symptoms will not be treated as adverse events as these are related to the outcome of the meta-analysis.

Related to objective (3), outcomes for prognostic factors at baseline will be associated with disease course of UTI in women allocated to experimental strategies. The outcomes will be: (a) recovery without antibiotics 
within 14 days (b) subsequent antibiotic treatment within at least 14 days (due to persistent/worsening symptoms, symptom relapse) (c) complications (eg, febrile UTI, pyelonephritis). Candidate prognostic factors and moderators will include age, presence, severity and duration of patient-reported symptoms (eg, dysuria, frequency, urgency, lower abdominal pain), physician-reported physical examination findings (eg, fever, loin pain), point-ofcare tests (eg, dipstick test results, C reactive protein, urine sediment, other point-of-care tests) and other additional variables like the bladder incubation time, at the initial consultation or inclusion, if available. ${ }^{20}$ Although urine culture results are not available at the time of the treatment decisions, we will investigate the results (positive or negative for the presence of uropathogens and antimicrobial resistance) as possible antibiotic effect moderators.

\section{Study designs}

RCTs will be included in the meta-analysis.

\section{Search strategy}

A comprehensive literature search will be conducted in the following databases: MEDLINE, EMBASE, Web of Science, Latin American and Caribbean Health Sciences Literature (LILACS), the Cochrane Database of Systematic Reviews, the Cochrane Central Register of Controlled Trials, the Health Technology Assessment Database (HTA) at the Centre for Reviews and Dissemination and ClinicalTrials.gov.

Publications from 1990 onwards will be considered. If feasible, results will be filtered for 'Clinical Study', and the search will be repeated without filter, but with publication dates from 2018 onwards to retrieve studies that have only recently been added to the database and may not be completely indexed. The search terms including relevant Medical Subject Headings and keywords are (urinary tract infection OR urinary tract infections OR UTI OR bacteriuria OR pyuria OR cystitis OR pyelonephritis) AND (antibiotic OR antibiotics OR anti-bacterial agents OR anti-microbial). Detailed search strategies for each literature database can be found in tables 1-6 and under the Search strategies: additional databases section.

Reference lists of included studies and relevant reviews will be checked to identify any additional relevant articles that were not captured by the search.

\section{Search strategies: additional databases}

Latin American and Caribbean Health Sciences Literature

(tw: ( (mh: 'urinary tract infections' OR "urinary tract infection" OR "urinary tract infection*" OR ("urinary" AND "tract" AND "infection*") OR "uti" OR mh: "bacteriuria" OR "bacteriuria" OR mh: "pyuria" OR "pyuria" OR mh: "cystitis" OR "cystitis" OR mh: "pyelonephritis" OR "pyelonephritis"))) AND (tw: ((mh: "anti-bacterial agents" OR ("anti-bacterial" AND agents) OR "anti-bacterial agents" OR mh: "antibiotic agent" OR "antibiotic*" OR "anti-microbial" OR ("anti" AND "microbial")))) AND (tw: ((mh: "random allocation" OR ("random" AND
Table 1 PubMed-search strategy: first search with filter 'Clinical Study' and '1990/01/01 to 2019/12/31'

\begin{tabular}{|c|c|}
\hline Search & Query \\
\hline 1 & $\begin{array}{l}\text { "urinary tract infections"[MeSH Terms] OR } \\
\text { ("urinary"[All Fields] AND "tract"[All Fields] } \\
\text { AND "infections"[All Fields]) OR "urinary tract } \\
\text { infections"(All Fields) }\end{array}$ \\
\hline 2 & $\begin{array}{l}\text { (“urinary”[All Fields] AND "tract”[All Fields] } \\
\text { AND “infection”[All Fields]) OR "urinary tract } \\
\text { infection"[All Fields] }\end{array}$ \\
\hline 3 & UTI[All Fields] \\
\hline 4 & $\begin{array}{l}\text { "bacteriuria"[MeSH Terms] OR "bacteriuria”[All } \\
\text { Fields] }\end{array}$ \\
\hline 5 & “pyuria”[MeSH Terms] OR “pyuria”[All Fields] \\
\hline 6 & "cystitis"[MeSH Terms] OR “cystitis"[All Fields] \\
\hline 7 & $\begin{array}{l}\text { "pyelonephritis"[MeSH Terms] OR } \\
\text { "pyelonephritis"[All Fields] }\end{array}$ \\
\hline 8 & \#1 OR \#2 OR \#3 OR \#4 OR \#5 OR \#6 OR \#7 \\
\hline 9 & $\begin{array}{l}\text { "anti-bacterial agents"[Pharmacological Action] } \\
\text { OR "anti-bacterial agents"[MeSH Terms] OR } \\
\text { ("anti-bacterial”[All Fields] AND "agents"[All } \\
\text { Fields]) OR "anti-bacterial agents"[All Fields] }\end{array}$ \\
\hline 10 & "antibiotic"[All Fields] OR "antibiotics"[All Fields] \\
\hline 11 & $\begin{array}{l}\text { "anti-microbial” [All Fields] OR (“anti” [All Fields] } \\
\text { AND "microbial” [All Fields]) }\end{array}$ \\
\hline 12 & \#9 OR \#10 OR \#11 \\
\hline 13 & \#8 AND \#12 \\
\hline 14 & \#8 AND \#12 Filters: Clinical Study \\
\hline 15 & $\begin{array}{l}\text { \#8 AND \#12 Filters: Clinical Study; Publication } \\
\text { date from 1990/01/01 to 2019/12/31 }\end{array}$ \\
\hline
\end{tabular}

MeSH, Medical Subject Headings.

"allocation") OR "random allocation" OR "randomised" OR "randomized" OR "controlled" OR "Clinical"))) AND (tw: ( (mh: "clinical trials as a topic" OR ("clinical" AND "trial”) OR "clinical trial" OR "trial" OR "study" OR mh: "randomized controlled trials as a topic"))) AND (instance:"regional") AND (db:("LILACS"))

HTA Database of the Center for Reviews and Dissemination (limitation 1990-2019)

((urinary tract infection*) AND (bacteriuria) OR (antibiotic*)) and (Project record:ZDT OR Full publication record:ZDT) IN HTA FROM 1990 TO 2019

ClinicalTrials.gov (applied filters: Interventional, Female, Adult (18-64), Older Adult (65+))

(Urinary Tract Infections OR bacteriuria OR pyuria OR cystitis OR pyelonephritis) AND (antibiotics OR antibiotic OR anti-bacterial OR anti-microbial)

\section{Study selection and quality assessment}

Screening of the search results for relevant studies will be done by two reviewers independently. Title and abstract will be screened and studies that clearly do not meet the 
Table 2 PubMed-search strategy: additional search without filter 'Clinical Study' and from 2018 onwards (to retrieve studies that have only recently been added to the database and may not be completely indexed in PubMed)

\section{Search Query}

1 "urinary tract infections"[MeSH Terms] OR ("urinary"[All Fields] AND "tract"[All Fields] AND "infections"[All Fields]) OR "urinary tract infections"[All Fields]

2 ("urinary"[All Fields] AND "tract"[All Fields] AND "infection"[All Fields]) OR "urinary tract infection"[All Fields]

\begin{tabular}{|c|c|}
\hline 3 & UTI(All Fields) \\
\hline 4 & $\begin{array}{l}\text { "bacteriuria"[MeSH Terms] OR "bacteriuria"[All } \\
\text { Fields] }\end{array}$ \\
\hline 5 & "pyuria"[MeSH Terms] OR "pyuria"[All Fields] \\
\hline 6 & "cystitis"[MeSH Terms] OR "cystitis"[All Fields] \\
\hline 7 & $\begin{array}{l}\text { "pyelonephritis"[MeSH Terms] OR } \\
\text { "pyelonephritis"[All Fields] }\end{array}$ \\
\hline 8 & \#1 OR \#2 OR \#3 OR \#4 OR \#5 OR \#6 OR \#7 \\
\hline 9 & $\begin{array}{l}\text { "anti-bacterial agents"[Pharmacological Action] } \\
\text { OR "anti-bacterial agents"[MeSH Terms] OR ("anti- } \\
\text { bacterial"[All Fields] AND "agents"[All Fields]) OR } \\
\text { "anti-bacterial agents"[All Fields] }\end{array}$ \\
\hline 10 & "antibiotic”[All Fields] OR “antibiotics”[All Fields] \\
\hline 11 & $\begin{array}{l}\text { "anti-microbial”[All Fields] OR (“anti”[All Fields] AND } \\
\text { "microbial”[All Fields]) }\end{array}$ \\
\hline 12 & \#9 OR \#10 OR \#11 \\
\hline 13 & $\begin{array}{l}\text { "random allocation"[MeSH Terms] OR ("random"[All } \\
\text { Fields] AND "allocation"[All Fields]) OR "random } \\
\text { allocation"[All Fields] OR "randomised"[All Fields] } \\
\text { OR "randomised"[All Fields] OR "controlled"[All } \\
\text { Fields] OR "clinical"[All Fields] }\end{array}$ \\
\hline 14 & $\begin{array}{l}\text { ("clinical trials as topic"[MeSH Terms] OR } \\
\text { ("clinical”[All Fields] AND "trials"[All Fields] AND } \\
\text { "topic"[All Fields]) OR "clinical trials as topic"[All } \\
\text { Fields] OR "trial”[All Fields]) OR "study"[All Fields] }\end{array}$ \\
\hline 15 & \#13 AND \#14 \\
\hline 16 & $\begin{array}{l}\text { "randomised controlled trials as topic"[MeSH } \\
\text { Terms] }\end{array}$ \\
\hline 17 & \#15 OR \#16 \\
\hline 18 & \#8 AND \#12 AND \#17 \\
\hline 19 & $\begin{array}{l}\text { \#8 AND \#12 AND \#19 Filters: Publication date from } \\
2018 / 01 / 01 \text { to } 2019 / 12 / 31\end{array}$ \\
\hline
\end{tabular}

$\mathrm{MeSH}$, Medical Subject Headings.

inclusion criteria will be excluded. Any disagreements and all remaining studies will be rescreened by a third reviewer. For all studies still remaining, full texts will be reviewed according to the pre-specified inclusion criteria by the third reviewer. The decision about inclusion or exclusion of a study will be discussed with another independent reviewer. RCTs in uncomplicated acute UTI in adult (adolescent) female patients presenting in general
Table 3 EMBASE-search strategy: first search with filter 'Controlled Clinical Trial/Randomized Controlled Trial' and ' $1990 / 01 / 01$ to $2019 / 12 / 31$ '

\begin{tabular}{|c|c|}
\hline Search & Query \\
\hline 1 & $\begin{array}{l}\text { 'urinary tract infection'/exp OR (urinary AND tract } \\
\text { AND infection*) OR 'urinary tract infection' }\end{array}$ \\
\hline 2 & uti \\
\hline 3 & 'bacteriuria'/exp OR bacteriuria \\
\hline 4 & 'pyuria'/exp OR pyuria \\
\hline 5 & 'cystitis'/exp OR cystitis \\
\hline 6 & 'pyelonephritis'/exp OR pyelonephritis \\
\hline 7 & \#1 OR \#2 OR \#3 OR \#4 OR \#5 OR \#6 \\
\hline 8 & $\begin{array}{l}\text { antiinfective agent'/exp OR ('anti-bacterial' AND } \\
\text { agent }^{\star} \text { ) }\end{array}$ \\
\hline 9 & 'antibiotic agent'/exp OR 'antibiotic*' \\
\hline 10 & ‘anti-microbial’ OR (anti AND microbial) \\
\hline 11 & \#8 OR \#9 OR \#10 \\
\hline 12 & \#7 AND \#11 \\
\hline 13 & $\begin{array}{l}\text { \#12 AND ('controlled clinical trial'/de OR } \\
\text { 'randomized controlled trial'/de OR 'randomized } \\
\text { controlled trial (topic)'/de) }\end{array}$ \\
\hline 14 & $\begin{array}{l}\text { \#12 AND ('controlled clinical trial'/de OR } \\
\text { 'randomized controlled trial'/de OR 'randomized } \\
\text { controlled trial (topic)'/de) AND(1990-2019)/py }\end{array}$ \\
\hline 15 & $\begin{array}{l}\text { \#12 AND ('controlled clinical trial'/de OR } \\
\text { 'randomized controlled trial'/de OR 'randomized } \\
\text { controlled trial (topic)'/de) AND(1990-2019)/py AND } \\
\text { (embase)/lim }\end{array}$ \\
\hline 16 & $\begin{array}{l}\text { \#12 AND ('controlled clinical trial'/de OR } \\
\text { 'randomized controlled trial'/de OR 'randomized } \\
\text { controlled trial (topic)'/de) AND(1990-2019)/py AND } \\
\text { (medline)/lim }\end{array}$ \\
\hline 17 & \#14 NOT \#16 \\
\hline
\end{tabular}

practice with any intervention to reduce antibiotic treatment and (immediate) antibiotic treatment as control will be included. Conference abstracts will be excluded. If appropriate, IPD of trials that do not compare directly an intervention to reduce antibiotic treatment with (immediate) antibiotic treatment can be considered in explorative analyses to strengthen, if only indirectly, the outlined comparisons.

Study quality will be assessed independently by two reviewers using the Cochrane Collaboration's tool for assessing risk of bias focusing on sequence generation and allocation sequence concealment (selection bias), blinding of participants and personnel (performance bias), blinding of outcome assessment (detection bias), incomplete outcome data (attrition bias) and selective outcome reporting (reporting bias). ${ }^{21}$ The potential for publication bias will be assessed using funnel plots (with and without studies lacking IPD) and corresponding asymmetry tests. To assess data availability bias characteristics 
Table 4 EMBASE-search strategy: additional search without filter 'Controlled Clinical Trial/Randomized Controlled Trial' and from 2018 onwards (to retrieve studies that have only recently been added to the database and may not be completely indexed in EMBASE)

\begin{tabular}{|c|c|}
\hline Search & Query \\
\hline 1 & $\begin{array}{l}\text { 'urinary tract infection'/exp OR (urinary AND tract } \\
\text { AND infection*) OR 'urinary tract infection' }\end{array}$ \\
\hline 2 & uti \\
\hline 3 & 'bacteriuria'/exp OR bacteriuria \\
\hline 4 & ‘pyuria’/exp OR pyuria \\
\hline 5 & 'cystitis'/exp OR cystitis \\
\hline 6 & 'pyelonephritis'/exp OR pyelonephritis \\
\hline 7 & \#1 OR \#2 OR \#3 OR \#4 OR \#5 OR \#6 \\
\hline 8 & $\begin{array}{l}\text { antiinfective agent'/exp OR ('anti-bacterial' AND } \\
\text { agent }^{\star} \text { ) }\end{array}$ \\
\hline 9 & ‘antibiotic agent’/exp OR ‘antibiotic`' \\
\hline 10 & ‘anti-microbial’ OR (anti AND microbial) \\
\hline 11 & \#8 OR \#9 OR \#10 \\
\hline 12 & $\begin{array}{l}\text { ‘randomization’/exp OR (random AND allocation) } \\
\text { OR randomi\$ed }\end{array}$ \\
\hline 13 & $\begin{array}{l}\text { 'clinical trial'/exp OR 'clinical study'/exp OR } \\
\text { (clinical AND trial) OR (clinical AND study) OR } \\
\text { 'controlled study'/exp OR 'controlled study' }\end{array}$ \\
\hline 14 & \#12 AND \#13 \\
\hline 15 & 'randomized controlled trial'/exp AND topic \\
\hline 16 & \#14 OR \#15 \\
\hline 17 & \#7 AND \#11 AND \#16 \\
\hline 18 & \#7 AND \#11 AND \#16 AND(2018-2019)/py \\
\hline 19 & $\begin{array}{l}\text { \#7 AND \#11 AND \#16 AND(2018-2019)/py AND } \\
\text { (medline)/lim }\end{array}$ \\
\hline 20 & \#18 NOT \#19 \\
\hline
\end{tabular}

of the studies with IPD and those without IPD will be compared. $^{22}$

\section{Data extraction and IPD collection}

For the IPD-meta-analysis anonymised data on individual patient level will be requested either in electronic form via standardised data extraction sheets and will be formatted in a consistent way to permit reanalysis. As the rating scales vary across some of the studies, the defined symptom severity for the primary and secondary outcomes may need to be rescaled. Data (baseline characteristics, outcomes and study characteristics) will be checked for accordance with the published data. If data appear inconsistent or unclear, this will be resolved by queries to the investigators. The investigators of currently nine available studies have been contacted in advance via personnel communication and agreed to provide IPD of their trials. Authors of further studies, which will be identified during the systematic literature search, will be contacted via email and asked to participate and to provide IPD of their
Table 5 Cochrane Library (CENTRAL and CDSR) search strategy (limitation 1990-2019)

\begin{tabular}{cl}
\hline Search & Query (all text) \\
\hline 1 & 'urinary tract infection' \\
2 & 'urinary tract infections' \\
3 & uti \\
4 & 'bacteriuria' \\
5 & 'pyuria' \\
6 & 'cystitis' \\
7 & 'pyelonephritis' \\
8 & \#1 OR \#2 OR \#3 OR \#4 OR \#5 OR \#6 OR \#7 \\
9 & 'anti-bacterial agents' \\
10 & 'antibiotic' \\
11 & 'anti-microbial' \\
12 & \#9 OR \#10 OR \#11 \\
13 & \#8 AND \#12 \\
\hline
\end{tabular}

CDSR, Cochrane Database of Systematic Reviews; CENTRAL, Cochrane Central Register of Controlled Trials.

trials. A comprehensive list of the requested variables are in the online supplemental file 2. If no IPD are accessible, aggregated data on baseline characteristics, treatments, symptoms and outcomes measures will be extracted from the publication in standardised forms by two reviewers independently.

Table 6 Web of Science-search strategy (limitation: article; 1990-2019)

\section{Search Query (all text)}

$1 \quad \mathrm{ALL}=$ ("urinary tract infection"” OR UTI OR bacteriuria OR pyuria OR cystitis OR pyelonephritis) Indexes=SCI-EXPANDED Timespan=All years

$2 \quad \mathrm{ALL}=$ (antibiotic* OR anti-microbial OR antibacterial) Indexes=SCI-EXPANDED Timespan=All years

$3 \quad$ \#2 AND \#1 Indexes=SCI-EXPANDED Timespan=All years

$4 \quad \mathrm{ALL}=$ ("random allocation") Indexes=SCI-EXPANDED Timespan=All years

$5 \quad \mathrm{ALL}=$ (randomized $\mathrm{OR}$ randomised $\mathrm{OR}$ controlled OR clinical) Indexes=SCI-EXPANDED Timespan=All years

$6 \quad \mathrm{ALL}=$ (trial OR study) Indexes $=$ SCI-EXPANDED Timespan=All years

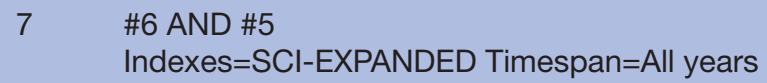

$8 \quad$ \#7 OR \#4 Indexes=SCI-EXPANDED Timespan=All years

$9 \quad$ \#8 AND \#3 Indexes=SCI-EXPANDED Timespan=All years

10 (\#8 AND \#3) AND DOCUMENT TYPES: (Article) Indexes=SCI-EXPANDED Timespan $=1990-2019$ 


\section{Analysis strategy and statistical methods}

Pairwise meta-analysis methods will be used for estimating treatment effects (relative to standard treatment) based on relevant subsets of studies. Meta-analysis will be carried out if at least two studies are available for comparison. In order to include effect moderators or prognostic factors, analyses are generalised to meta-regressions. In all cases, analyses will be stratified by incorporating studyspecific random effects, and, in a secondary analysis, possibly also centre-effects in IPD. IPD are considered via appropriate likelihoods (eg, binomial likelihoods for binary outcomes); study-level summary estimates (eg, log ORs) are included using an approximate normal likelihood (where appropriate). In a sensitivity analysis, we will also incorporate the IPD in terms of a summary estimate in a (technically simpler) two-stage meta-analysis. The investigation of prognostic factors (objective (3)) will require IPD. Prognostic factors will also be considered in terms of random effects, if supported by a better model fit, or otherwise as fixed effects. Missing data for certain patients in IPD will be imputed if necessary, given that the missing cases only make up a minority, and that these may plausibly be considered missing at random. ${ }^{23}$ The models used all fall into the general class of generalised linear mixed models, a special type of hierarchical model accounting for variability at the patient-level as well as the study-level. In order to include also indirect evidence on treatment effects, the feasibility of a (joint) network metaanalysis will be explored.

Primary analyses will focus on ORs as effect measures, but relative risks will be explored as an alternative. Analyses will be performed using Bayesian methods with uninformative priors for treatment effects and weakly informative priors for between-study variability (heterogeneity), as these are better suited for the case of few studies only. ${ }^{24}$ For objective (1), the variables urgency, frequency and dipstick results such as erythrocytes, leucocytes and nitrite will be included as main effects in the model. ${ }^{25-27}$ Other additional variables (like age, further UTI symptoms, fever, urine culture results, $\mathrm{C}$ reactive protein, other point-of-care tests) will be considered in a variable selection stage. Concerning objective (2), symptom duration, worsening of symptoms and relapse will be considered as potential treatment effect moderators. Effect estimates (ORs or relative risks) will be quoted along with two-sided 95\% credible intervals and posterior tail probabilities. Model building will be done using forward selection based on Bayes factors (if sensible and feasible), or approximations like information criteria (eg, the deviance information criterion). Besides pairwise comparisons of specific medications (eg, fosfomycin vs ibuprofen), groups of similar treatments will be grouped in broader categories (eg, first-line and second-line antibiotics, pain killers, herbal treatments, delayed prescription or placebo). Between-study variability will be visually explored in forest plots of (log) ORs and quantified using the SD parameter tau. Sensitivity of results to modelling approaches will be assessed for example, by varying prior assumptions for the heterogeneity, by considering subgroups of studies based on suitable geographical regions or by exclusion of individual studies based on quality criteria. The performance of prognostic models (objective (3)) will be assessed in an internal-external cross-validation fashion. ${ }^{23}$ While the validation of prognostic models is fairly established, the evaluation of prediction models (objectives (1) and (2)) is an area of ongoing research. ${ }^{28}$ For this purpose it may be possible to use, for example, a C-statistic approach. ${ }^{29}$

Descriptive summaries are used to describe studycharacteristics and patient-characteristics, including as complete as possible details on missing values. Metric variables are characterised by mean and SD (and range, where appropriate) ordinal scaled variables by median and percentiles. Discrete variables are summarised by quoting absolute or relative frequencies.

We will use the $\mathrm{R}$ software (including appropriate add-on packages, for example, LME, JAGS, STAN, metafor, bayesmeta, MetaStan) for all statistical analyses.

To rate the confidence in cumulative evidence, the strength of the body of evidence (quality or certainty of evidence and strength of recommendation) will be assessed using The Grading of Recommendations Assessment, Development and Evaluation framework. ${ }^{30}$

\section{Patient and public involvement}

Our research team is collaborating with a patient advisory board consisting of about eight citizens which has recently been established at the Department of General Practice of the University of Wuerzburg (see http://www.allgemeinmedizin.uni-wuerzburg.de/forschung/buergerforum/). The board has been introduced to discuss the designs of the department's scientific studies to ensure that they are comprehensible and relevant for humans. ${ }^{31}$ It has been invited to give feedback to the aims and outcomes of this review and will be involved to participate in development of the prognostic model. The group will also be involved as a patient advisory board of a practice-based research network in Bavaria (see http://www.allgemeinmedizin. uni-wuerzburg.de/forschung/forschungspraxennetz/).

\section{ETHICS AND DISSEMINATION}

The meta-analysis is based on previously published studies. The local institutional review and ethics board judged the project a secondary analysis of existing anonymous data which meet the criteria for waiver of ethics review. Data sharing statement according to current guidelines of the International Committee of Medical Journal Editors (ICMJE) will be required from authors providing IPD.

Dissemination of the results will be via published research papers in high-quality peer-reviewed open access journals and presentations at scientific meetings. Additionally, key messages will be promoted to clinicians and patients via direct mailings, communications via clinical research networks, social media, press releases or patient blogs. This work will aim to influence guidelines on 
clinical management of UTI symptoms both on national (national guidelines) and international levels.

\section{Author affiliations}

${ }^{1}$ Department of Medical Statistics, University Medical Center Goettingen, Goettingen, Germany

${ }^{2}$ Department of General Practice, University Medical Center Goettingen, Goettingen, Germany

${ }^{3}$ Department of Emergency Medicine, University Medical Center Goettingen, Goettingen, Germany

${ }^{4}$ Department of General Practice, Julius Maximilians University Wuerzburg Faculty of Medicine, Wuerzburg, Germany

${ }^{5}$ Julius Center for Health Sciences and Primary Care, University Medical Center Utrecht, Utrecht, The Netherlands

${ }^{6}$ Centre for Academic Primary Care, University of Bristol, Bristol, UK

${ }^{7}$ Department of Public Health and Primary Care, University of Ghent, Gent, Belgium

${ }^{8}$ Department of General Practice, The Antibiotic Centre of Primary Care, Institute of Health and Society, University of Oslo, Oslo, Norway

${ }^{9}$ Department of Emergency General Practice, Oslo Accident and Emergency Outpatient Clinic, Oslo, Norway

${ }^{10}$ Primary Care Population Sciences and Medical Education Unit, University of Southampton School of Medicine, Southampton, UK

${ }^{11}$ Clinic for Urology, Pediatric Urology and Andrology, Justus Liebig University

Giessen Faculty of Medicine, Giessen, Germany

${ }^{12}$ Institute for Infectious Diseases, University of Bern, Bern, Switzerland

${ }^{13}$ Department of Clinical Microbiology, Bacteriology, Umea University, Umea, Sweden

Twitter Thomas Debray @TPA_Debray, Sven Ferry @Sven Ferry and Tim Friede @ tim friede

Acknowledgements We are very grateful to Peter Jüni and Thierry Christiaens for their support in drafting the proposal of this project.

Contributors IG, TF, EH, ADH, MM, IV, PL, ML, SH, TM, SF, AK, BS and FW: conception of the study. TF, CR and TD: statistical concept. GF, YK, IG and JH: contact to possible cooperation partners. $\mathrm{CR}$ and $\mathrm{JH}$ : drafting the manuscript. CR and $\mathrm{JH}$ : developing search strategy. All authors: revising the manuscript critically for important intellectual content and approval of the final version of the manuscript. IG: guarantor of the review.

Funding This work is funded by the Federal Ministry of Education and Research (BMBF), research grant number KS2017-047. TPAD is funded by The Netherlands Organisation for Health Research and Development (grant 91617050).

Disclaimer The funders were not involved in developing the protocol.

Competing interests IG, GF, TF, EH, MM are involved in the following studies: 'REGATTA — reducing antibiotic use for uncomplicated urinary tract infection in general practice by treatment with uva-ursi'. Afshar K, Fleischmann N, Schmiemann G, Bleidorn J, Hummers-Pradier E, Friede T, Wegscheider K, Moore M, Gagyor I. 'Reducing antibiotic use for uncomplicated urinary tract infection in general practice by treatment with uva-ursi (REGATTA) — a double-blind, randomised, controlled comparative effectiveness trial'. BMC Complement Altern Med. $2018 \mathrm{Jul}$ 3;18(1):203. doi: 10.1186/s12906-018-2266-x). IV was involved in the study: 'Vik I, Bollestad M, Grude N, Bærheim A, Damsgaard E, Neumark T, Bjerrum L, Cordoba G, Olsen IC, Lindbæk M. Ibuprofen versus pivmecillinam for uncomplicated urinary tract infection in women — a double-blind, randomised non-inferiority trial'. PLOS Med 15;5:e1002569. Doi.org/10.1371/journal.pmed.1002569. MM, ADH and PL are coauthors of the study: 'Moore M, Trill J, Simpson C, Webley F, Radford M, Stanton L, Maishman T, Glanopoulou A, Flower A, Eyles C, Willcox M, Hay AD, van der Werf E, Gibbons S, Lewith G, Little P, Griffiths G. Uva-ursi extract and ibuprofen as alternative treatments for uncomplicated urinary tract infection in women (ATAFUTI): a factorial randomised trial. Clinical Microbiology and Infection'. Doi.org/10.1016/j. cmi.2019.01.011. SH was involved in the study with the reference number 11. PL and MM were involved in the study with the reference number 12. SF and TM were involved in the study with the reference number 13. IG, EH were involved in the studies with the reference numbers 14 and 15 . AK was involved in the study with the reference number 16 .

Patient and public involvement Patients and/or the public were involved in the design, or conduct, or reporting, or dissemination plans of this research. Refer to the Methods section for further details.

Patient consent for publication Not required.
Provenance and peer review Not commissioned; externally peer reviewed.

Open access This is an open access article distributed in accordance with the Creative Commons Attribution Non Commercial (CC BY-NC 4.0) license, which permits others to distribute, remix, adapt, build upon this work non-commercially, and license their derivative works on different terms, provided the original work is properly cited, appropriate credit is given, any changes made indicated, and the use is non-commercial. See: http://creativecommons.org/licenses/by-nc/4.0/.

\section{ORCID iDs}

Judith Heinz http://orcid.org/0000-0001-8331-9137

Ingvild Vik http://orcid.org/0000-0002-8947-2914

Paul Little http://orcid.org/0000-0003-3664-1873

Michael Moore http://orcid.org/0000-0002-5127-4509

Tim Friede http://orcid.org/0000-0001-5347-7441

Ildiko Gagyor http://orcid.org/0000-0002-7974-7603

\section{REFERENCES}

1 Laupland KB, Ross T, Pitout JDD, et al. Community-onset urinary tract infections: a population-based assessment. Infection 2007;35:150-3.

2 Butler CC, Hawking MKD, Quigley A, et al. Incidence, severity, help seeking, and management of uncomplicated urinary tract infection: a population-based survey. Br J Gen Pract 2015;65:e702-7.

3 Wagenlehner FME, Schmiemann G, Hoyme U, et al. [National S3 guideline on uncomplicated urinary tract infection: recommendations for treatment and management of uncomplicated communityacquired bacterial urinary tract infections in adult patients]. Urologe $A$ 2011:50:153-69.

4 Colgan R, Williams M. Diagnosis and treatment of acute uncomplicated cystitis. Am Fam Physician 2011;84:771-6.

5 Mclsaac WJ, Low DE, Biringer A, et al. The impact of empirical management of acute cystitis on unnecessary antibiotic use. Arch Intern Med 2002;162:600-5.

6 Foxman B. Urinary tract infection syndromes: occurrence, recurrence, bacteriology, risk factors, and disease burden. Infect Dis Clin North Am 2014;28:1-13.

7 Ong DSY, Kuyvenhoven MM, van Dijk L, et al. Antibiotics for respiratory, ear and urinary tract disorders and consistency among GPs. J Antimicrob Chemother 2008;62:587-92.

8 Hooton TM. Clinical practice. Uncomplicated urinary tract infection. N Engl J Med 2012;366:1028-37.

9 Willems CSJ, van den Broek D’Obrenan J, Numans ME, et al. Cystitis: antibiotic prescribing, consultation, attitudes and opinions. Fam Pract 2014;31:149-55.

10 Costelloe C, Metcalfe C, Lovering A, et al. Effect of antibiotic prescribing in primary care on antimicrobial resistance in individual patients: systematic review and meta-analysis. BMJ 2010;340:c2096.

11 Christiaens TCM, De Meyere M, Verschraegen G, et al. Randomised controlled trial of nitrofurantoin versus placebo in the treatment of uncomplicated urinary tract infection in adult women. $\mathrm{Br} J$ Gen Pract 2002;52:729-34.

12 Little P, Merriman R, Turner S, et al. Presentation, pattern, and natural course of severe symptoms, and role of antibiotics and antibiotic resistance among patients presenting with suspected uncomplicated urinary tract infection in primary care: observational study. BMJ 2010;340:b5633.

13 Ferry SA, Holm SE, Stenlund H, et al. Clinical and bacteriological outcome of different doses and duration of pivmecillinam compared with placebo therapy of uncomplicated lower urinary tract infection in women: the LUTIW project. Scand J Prim Health Care 2007;25:49-57

14 Bleidorn J, Gágyor I, Kochen MM, et al. Symptomatic treatment (ibuprofen) or antibiotics (ciprofloxacin) for uncomplicated urinary tract infection?-results of a randomized controlled pilot trial. BMC Med 2010;8:30.

15 Gágyor I, Bleidorn J, Kochen MM, et al. Ibuprofen versus fosfomycin for uncomplicated urinary tract infection in women: randomised controlled trial. BMJ 2015;351:h6544.

16 Kronenberg A, Bütikofer L, Odutayo A, et al. Symptomatic treatment of uncomplicated lower urinary tract infections in the ambulatory setting: randomised, double blind trial. BMJ 2017;359:j4784.

17 Moher D, Liberati A, Tetzlaff J, et al. Preferred reporting items for systematic reviews and meta-analyses: the PRISMA statement. PLoS Med 2009;6:e1000097.

18 Vik I, Bollestad M, Grude N, et al. Ibuprofen versus pivmecillinam for uncomplicated urinary tract infection in women-A double-blind, randomized non-inferiority trial. PLoS Med 2018;15:e1002569. 
19 Bleidorn J, Hummers-Pradier E, Schmiemann G, et al. Recurrent urinary tract infections and complications after symptomatic versus antibiotic treatment: follow-up of a randomised controlled trial. Ger Med Sci 2016;14:Doc01.

20 Ferry SA, E Holm S, Ferry BM, et al. High diagnostic accuracy of nitrite test paired with urine sediment can reduce unnecessary antibiotic therapy. Open Microbiol J 2015;9:150-9.

21 Higgins J, Green S. Cochrane handbook for systematic reviews of interventions version 5.1.0., chapter 8 . The Cochrane collaboration, 2011. Available: www.handbook.cochrane.org

22 Ahmed I, Sutton AJ, Riley RD. Assessment of publication bias, selection bias, and unavailable data in meta-analyses using individual participant data: a database survey. BMJ 2012;344:d7762.

23 Audigier V, White IR, Jolani S, et al. Multiple imputation for multilevel data with continuous and binary variables. Stat Sci 2018;33:160-83.

24 Friede T, Röver C, Wandel S, et al. Meta-analysis of few small studies in orphan diseases. Res Synth Methods 2017;8:79-91.

25 Gágyor I, Haasenritter J, Bleidorn J, et al. Predicting antibiotic prescription after symptomatic treatment for urinary tract infection: development of a model using data from an RCT in general practice. Br J Gen Pract 2016;66:e234-40.
26 Mclsaac WJ, Moineddin R, Ross S. Validation of a decision aid to assist physicians in reducing unnecessary antibiotic drug use for acute cystitis. Arch Intern Med 2007;167:2201-6.

27 Little P, Turner S, Rumsby K, et al. Dipsticks and diagnostic algorithms in urinary tract infection: development and validation, randomised trial, economic analysis, observational cohort and qualitative study. Health Technol Assess 2009;13:1-73.

28 Kent DM, Steyerberg E, van Klaveren D. Personalized evidence based medicine: predictive approaches to heterogeneous treatment effects. BMJ 2018;363:k4245.

29 van Klaveren D, Steyerberg EW, Serruys PW, et al. The proposed 'concordance-statistic for benefit' provided a useful metric when modeling heterogeneous treatment effects. $J$ Clin Epidemiol 2018;94:59-68.

30 Atkins D, Best D, Briss PA, et al. Grading quality of evidence and strength of recommendations. BMJ 2004;328:1490.

31 Schilling I. Was wünschen sich Patientinnen bei Der Behandlung von Harnwegsinfekten? - eine Fokusgruppenanalyse 50 Kongress für Allgemeinmedizin und Familienmedizin, 2016. 